\title{
Thermal Properties Measurement Report
}

PEMP 1.1.B Team:

Jon Carmack, Lori Braase, Cynthia Papesch, David Hurley, Michael Tonks, Yongfeng Zhang, Krzysztof Gofryk, Jason Harp, Randy Fielding, Collin Knight, and Mitch Meyer

\section{August 2015}

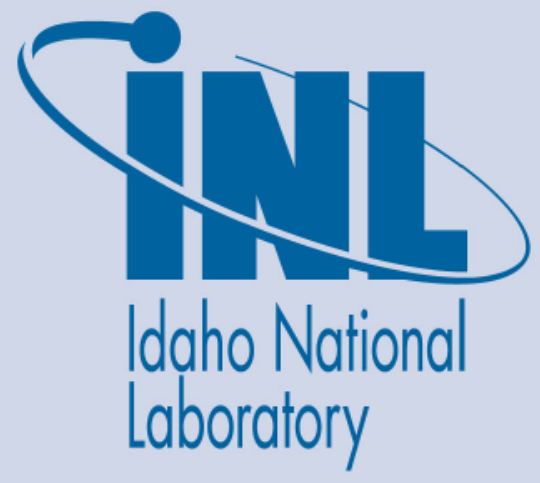

The INL is a U.S. Department of Energy National Laboratory operated by Battelle Energy Alliance 


\section{DISCLAIMER}

This information was prepared as an account of work sponsored by an agency of the U.S. Government. Neither the U.S. Government nor any agency thereof, nor any of their employees, makes any warranty, expressed or implied, or assumes any legal liability or responsibility for the accuracy, completeness, or usefulness, of any information, apparatus, product, or process disclosed, or represents that its use would not infringe privately owned rights. References herein to any specific commercial product, process, or service by trade name, trade mark, manufacturer, or otherwise, does not necessarily constitute or imply its endorsement, recommendation, or favoring by the U.S. Government or any agency thereof. The views and opinions of authors expressed herein do not necessarily state or reflect those of the U.S. Government or any agency thereof. 
INL/EXT-15-36283

Revision 0

\title{
Thermal Properties Measurement Report
}

\author{
PEMP 1.1.B Team: \\ Jon Carmack, Lori Braase, Cynthia Papesch, David Hurley, Michael Tonks, \\ Yongfeng Zhang, Krzysztof Gofryk, Jason Harp, Randy Fielding, Collin Knight, \\ and Mitch Meyer
}

August 2015

Idaho National Laboratory Idaho Falls, Idaho $\mathbf{8 3 4 1 5}$

http://www.inl.gov

\author{
Prepared for the \\ U.S. Department of Energy \\ Office of Nuclear Energy \\ Under DOE Idaho Operations Office \\ Contract DE-AC07-05ID14517
}





\section{SUMMARY}

The FY2015 Performance Evaluation and Measurement Plan (PEMP), by which Battelle Energy Alliance is evaluated by the U.S. Department of Energy (DOE), includes a section that is focused on the development of thermal property measurement capability on fresh and irradiated nuclear fuels and materials at Idaho National Laboratory (INL). The goals are stated as:

Develop fuel thermal conductivity measurement capability. Demonstrate a new science-based approach to fuels development by performing thermal conductivity measurements on advanced fuel samples (beyond $\mathrm{UO}_{2}$ ). Demonstrate how thermal property measurements can be taken on Accident Tolerant Fuel (ATF) concepts to support DOE's evaluation of ATF concepts. FY 2015 scope is limited to capability demonstration using un-irradiated or surrogate samples and does not include measurements on irradiated fuel.

A new scientific approach and methodology was developed for studying nuclear fuel system thermal properties that will be used for future nuclear fuels and materials research and development at the Idaho National Laboratory. This science-based method connects advanced multi-scale, multi-physics modeling with multi-scale experimental measurements significantly improving the understanding of advanced nuclear fuel system performance and behavior. It is expected that this methodology will be adopted and utilized by leading research institutions in the field.

The Thermal Properties Measurement Report summarizes the research, development, installation, and initial use of significant experimental thermal property characterization capabilities at the INL in FY 2015. These new capabilities were used to characterize a $\mathrm{U}_{3} \mathrm{Si}_{2}$ (candidate Accident Tolerant) fuel sample fabricated at the INL. The ability to perform measurements at various length scales are important and provide additional data that is not currently in the literature. However, the real value of the data will be in accomplishing a phenomenological understanding of the thermal conductivity in fuels and the ties to predictive modeling. Thus, the MARMOT advanced modeling and simulation capability was utilized to illustrate how the microstructural data can be modeled and compared with bulk characterization data. A scientific method was established for thermal property measurement capability on irradiated nuclear fuel samples, which will be installed in the Irradiated

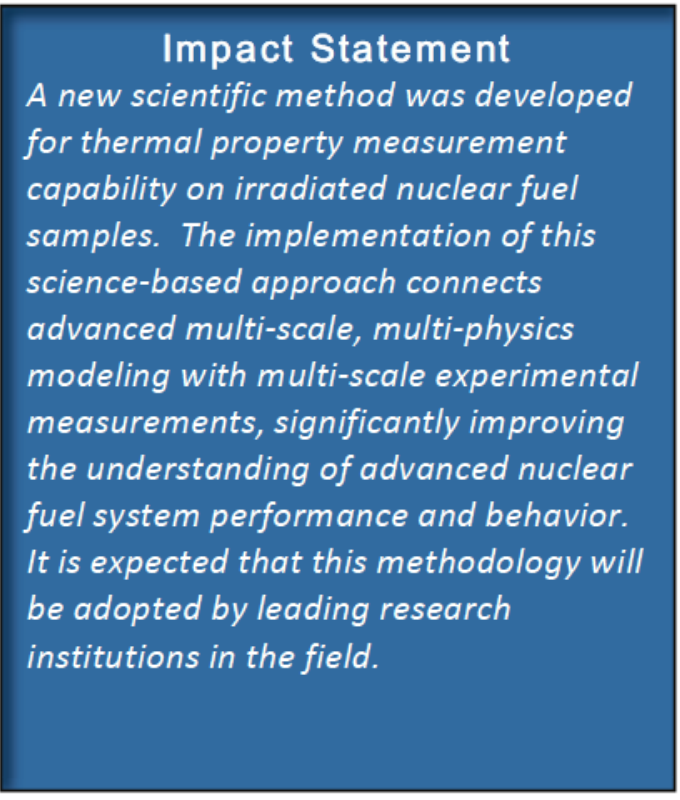
Material Characterization Laboratory (IMCL). 

August 11, 2015 


\section{CONTENTS}

Summary iii

Acronyms vii

Introduction 1

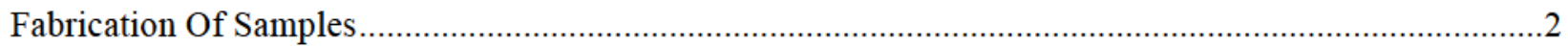

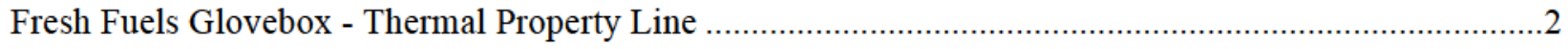

Laser Based Thermal Property Measurement ……...........................................................................

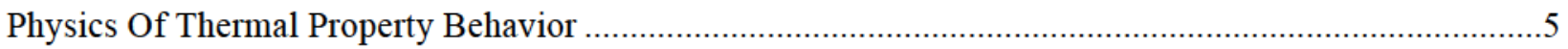

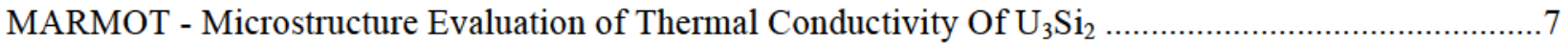

IMCL Thermal Property Measurement Capability Strategy ..............................................................

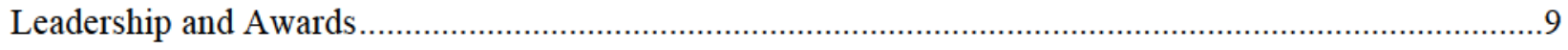

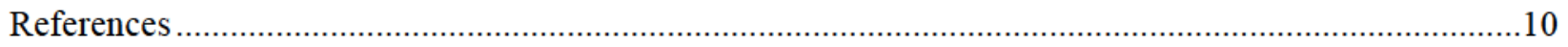

FIGURES

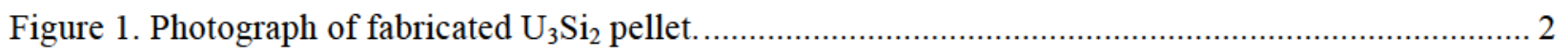

Figure 2. SEM photomicrograph of the $\mathrm{U}_{3} \mathrm{Si}_{2}$ as-fabricated pellet microstructure ................................... 2

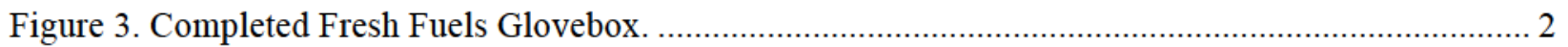

Figure 4. Netzsch Laser Flash Analyzer (LFA427) ....................................................................... 3

Figure 5. Thermal diffusivity vs. Temperature for $\mathrm{U}_{3} \mathrm{Si}_{2}$ using Pulse Laser Flash.................................. 3

Figure 6. Density vs. Temperature for $\mathrm{U}_{3} \mathrm{Si}_{2}$ Calculated from Coefficient of Thermal Expansion.............. 4

Figure 7. Left: Best fit results for $\mathrm{DU}_{3} \mathrm{Si}_{2}$ sample. Dots correspond to data and solid lines correspond to best fit modeling results. Right: Slope of the phase profile $(q)$ in the far field of the source versus frequency. There is good sensitivity to both $k_{s}$ and $D_{s}$ for the frequency range investigated.

Figure 8. Quantum Design DynaCool-9 measurement system (IRC, IF-603, C6). This closed circle measurement platform allows variety of transport and thermodynamic measurements of nuclear materials in wide temperature and magnetic field ranges.

Figure 9. Total thermal conductivity measured on bulk $\mathrm{U}_{3} \mathrm{Si}_{2}$ sample in the range from $2 \mathrm{~K}$ to $350 \mathrm{~K}$. Inset: experimental setup used for the thermal conductivity measurements in the DynaCool system.

Figure 10. (a) SEM image of a $\mathrm{U}_{3} \mathrm{Si}_{2}$ sample, (b) reconstructed microstructure and mesh in MOOSE for MARMOT calculations, and (c) thermal conductivity of U3Si2 as a function of temperature. The solids symbols are experimental measurements from literature, INL Thermal Flash, INL TCM, and INL PPMS experimental as indicated. 
The MARMOT results based on the reconstructed mesh are represented by the open triangles at $300 \mathrm{~K}$ 


\section{ACRONYMS}

AFC Advanced Fuels Cycle

ATF Accident Tolerant Fuel

DOE U.S. Department of Energy

FCRD Fuel Cycle Research and Development

FFG Fresh Fuels Glovebox

FOA Funding Opportunity Announcement

IMCL Irradiated Material Characterization Laboratory

INL Idaho National Laboratory

MBM MOOSE-BISON-MARMOT (code system)

MFC Materials and Fuels Complex

PEMP Performance Evaluation and Measurement Plan

PPMS physical properties measurement system

SEM Scanning Electron Microscope

TCM Thermal Conductivity Microscope

WEC Westinghouse Electric Company 

August 11, 2015 


\section{Thermal Properties Measurement Report INTRODUCTION}

Thermal properties of nuclear fuels and materials are key attributes for understanding and predicting the performance of a nuclear fuels and materials system. Thermal properties must be known on both fresh and irradiated fuels across temperature and material variations and are the key set of material properties that control how heat is transferred from a nuclear fuel to surrounding coolant. Thermal properties determine the fuel pin peak operating power, margin to melting, and fuel pin design and have a large bearing on fuel performance. Empirical data on thermal properties as a function of temperature and burnup can directly reduce design conservatism.

This data is used to validate mesoscale models. Understanding of the effect of microstructural features such as grain boundaries and impurity phases on thermal properties can be gained by making measurement of properties at the bulk and single grain levels. Fundamental understanding of thermal transport mechanisms is used to validate simulations at the atomic scale. Data at all scales is used to validate fuel behavior models, which are implemented in fuel performance codes that can be used to extrapolate fuel behavior outside of the test environment or test fuel microstructure, reducing the size, cost and time required for experimental testing and providing higher confidence in fuel design.

Idaho National Laboratory (INL) and associated nuclear fuels research and development programs have invested heavily over the years in infrastructure and capability development associated with measuring and determining material properties The opportunity to develop thermal property measurement capability was identified as a focus activity with near term demonstrations. This new capability fits into the plans for future development of fresh and irradiated nuclear fuels, especially with respect to establishing the appropriate thermal property measurement capability in the Irradiated Materials Characterization Laboratory (IMCL).

INL accomplished and exceeded the FY 2015 goals by establishing the capability to measure thermal properties of radioactive minor actinide and transuranic bearing samples, demonstrating thermal properties measurements on advanced nuclear fuel systems, leading research in the field of thermal property measurements, and paving a path for the future of thermal property science at INL. The capability is fully established to measure thermal properties on fresh fuels, including fuels containing radioactive and toxic materials, in the Thermal Properties Glovebox in the Analytical Laboratory.

To demonstrate this capability, an Accident Tolerant Fuel (ATF) sample of $\mathrm{U}_{3} \mathrm{Si}_{2}$ (Uranium Silicide) was fabricated at INL on behalf of Westinghouse Electric Company (WEC) under the U.S. Department of Energy (DOE) Funding Opportunity Announcement (FOA) industry program. The bulk thermal properties of the sample were characterized in the thermal properties glovebox and then the micro-scale thermal properties were characterized using the Thermal Conductivity Microscope and the new sub-room temperature thermal properties laboratory. Characterization using all of these methods yielded the data necessary to connect the microstructure level material properties with the bulk material properties, a capability demonstrating a "Science-based approach" to fuel research and development. The MOOSEBISON-MARMOT (MBM) code system was utilized to make the connection analytically between the microstructural level material properties measured using the Thermal Conductivity Microscope (TCM) and the bulk properties of the fuel system using the capabilities in the fresh fuels glovebox.

Finally, the path forward for measurement of thermal properties on irradiated nuclear fuel samples was established by demonstrating the capability to calculate the thermal properties at bulk scale using microstructural level measurements and modeling and simulation using the MARMOT simulation capability of MBM. 


\section{FABRICATION OF SAMPLES}

The INL teamed with WEC in development of $\mathrm{U}_{3} \mathrm{Si}_{2}$ based fuel. A high density fuel system, $\mathrm{U}_{3} \mathrm{Si}_{2}$, has the potential to provide significant performance enhancement over $\mathrm{UO}_{2}$ due to higher thermal conductivity of the $\mathrm{U}_{3} \mathrm{Si}_{2}$ composition. One of the WEC pellets fabricated at INL at the Materials and Fuels Complex (MFC) was used to demonstrate the capability to measure the thermal properties of candidate accident tolerant fuel compositions. Figure 1 shows a photograph of the pellet and Figure 2 is a scanning electron microscope (SEM) photomicrograph showing the microstructure of the $\mathrm{U}_{3} \mathrm{Si}_{2}$ asfabricated pellet.

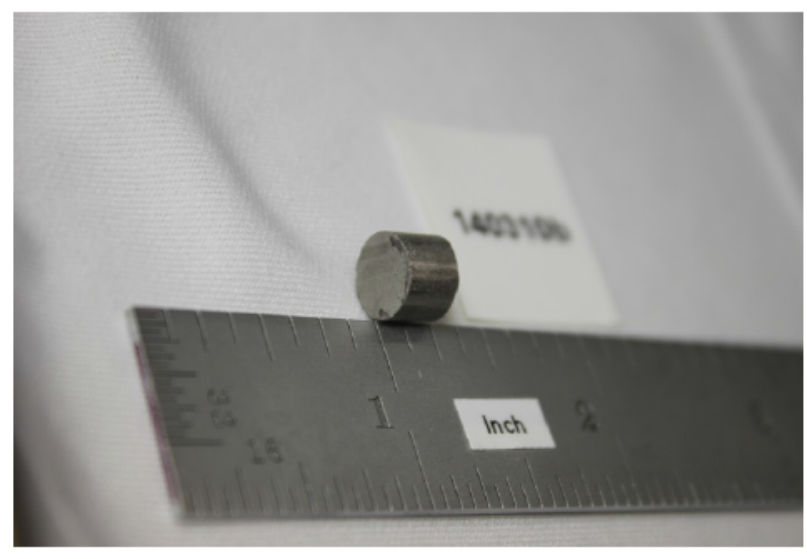

Figure 1. Photograph of fabricated $\mathrm{U}_{3} \mathrm{Si}_{2}$ pellet.

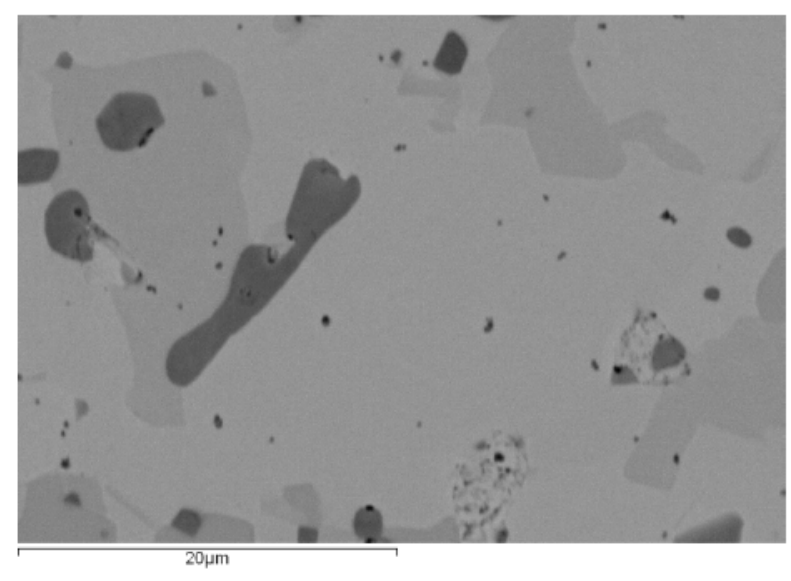

Figure 2. SEM photomicrograph of the $\mathrm{U}_{3} \mathrm{Si}_{2}$ asfabricated pellet microstructure.

\section{FRESH FUELS GLOVEBOX - THERMAL PROPERTY LINE}

A major activity for INL and the Fuel Cycle Research and Development (FCRD) Advanced Fuels Campaign (AFC) over the past few years has been the construction of the new thermal properties characterization capability in the Analytical Laboratory, referred to as the "Fresh Fuels Glovebox" (FFG) (Figure 3. Completed Fresh Fuels Glovebox.).
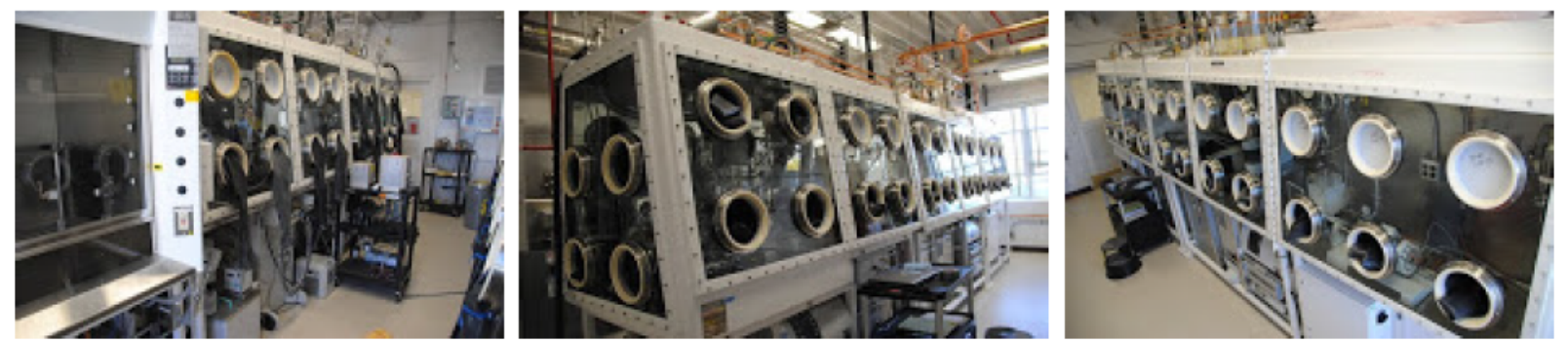

Figure 3. Completed Fresh Fuels Glovebox.

A key feature is full bulk thermal property measurement capability in an inert environment for asfabricated nuclear fuels and materials. The glovebox and thermal property characterization line became fully operational and was used to measure the bulk thermal properties of an $\mathrm{U}_{3} \mathrm{Si}_{2}$ fuel sample, a potential ATF technology. 
Thermal diffusivity measurements were conducted on a low enriched uranium silicide $\left(\mathrm{U}_{3} \mathrm{Si}_{2}\right)$ sample from room temperature to $1000^{\circ} \mathrm{C}$ to verify the pulse laser flash method for collecting thermal diffusivity data. These data were collected using the Netzsch Laser Flash Analyzer (LFA427) (Figure 4) modified for installation into the inert atmosphere FFG.

Figure 5 shows the thermal diffusivity vs. temperature for the $\mathrm{U}_{3} \mathrm{Si}_{2}$ material. The thermal diffusivity data is compared to data collected by White et.al, [1] on a similar material with higher density. INL data is lower than the literature data due to the lower density of the sample and/or secondary phase impurities, although both are known to result in an increase in thermal diffusivity with increasing temperature. Error bars were added to the data based upon the reported error ${ }^{\mathrm{i}}$ and the calculated error from measurements taken on a standard reference material. Figure 6 compares the density of the sample to that of the literature data. Density was calculated from the coefficient of linear thermal expansion in both the data and literature. The linear thermal expansion was measured using a pushrod dilatometer.

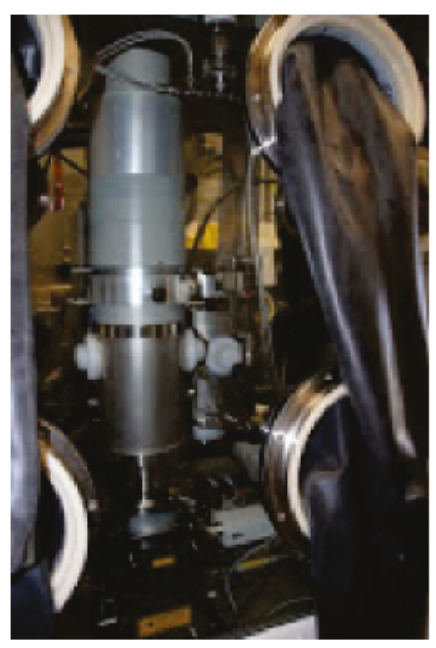

Figure 4. Netzsch Laser Flash Analyzer (LFA427).

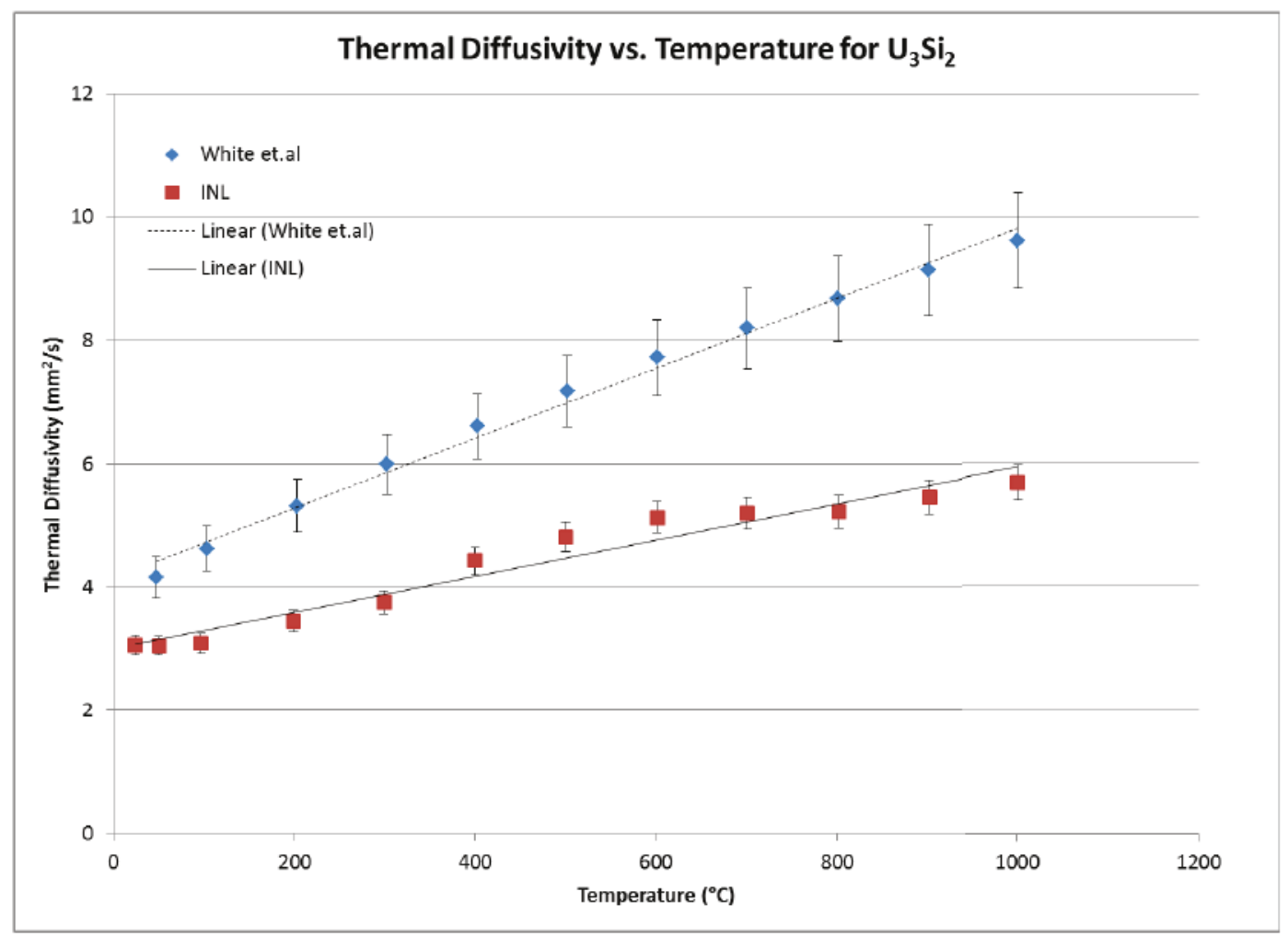

Figure 5. Thermal diffusivity vs. Temperature for $\mathrm{U}_{3} \mathrm{Si}_{2}$ using Pulse Laser Flash. 


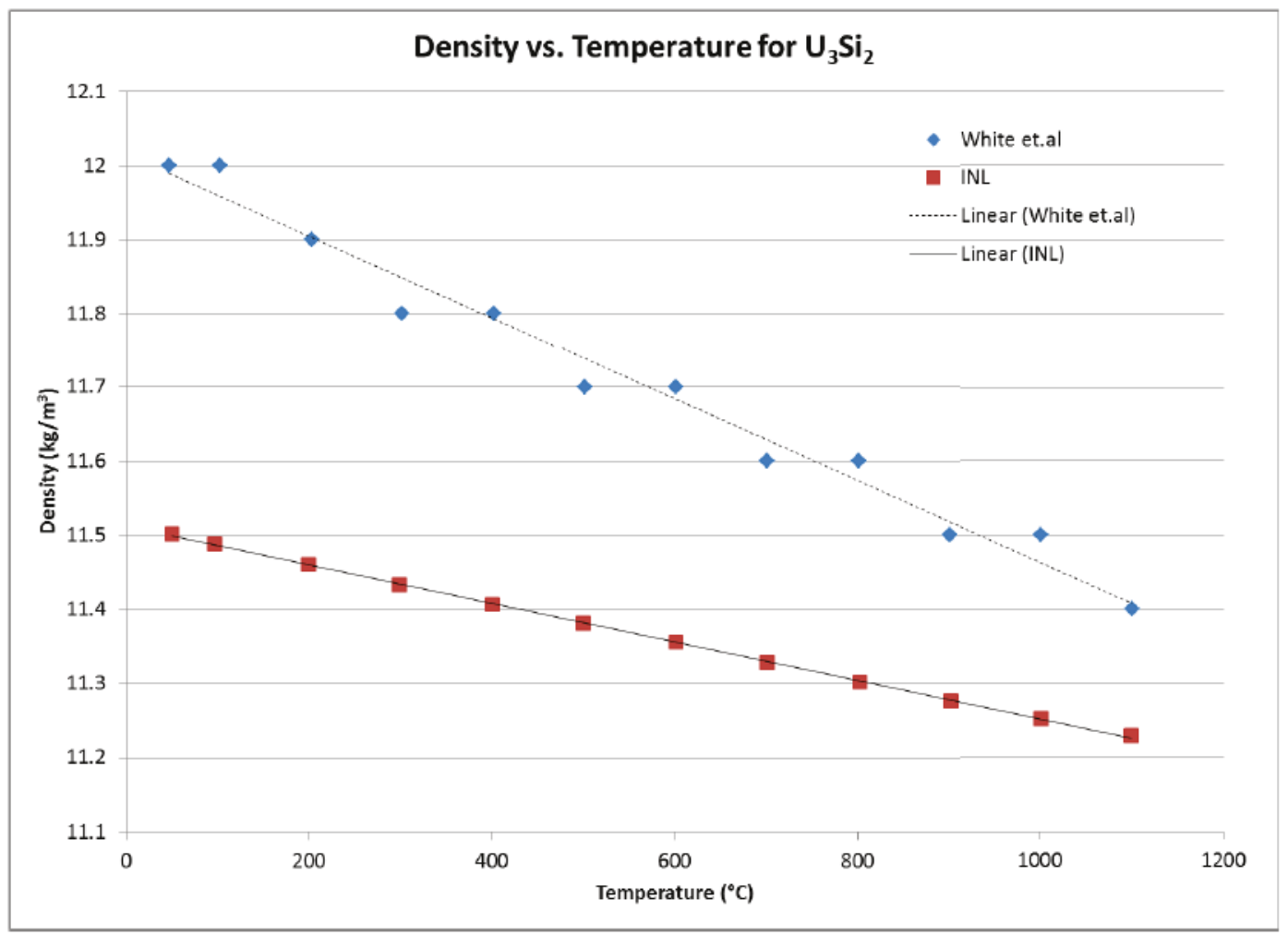

Figure 6. Density vs. Temperature for $\mathrm{U}_{3} \mathrm{Si}_{2}$ Calculated from Coefficient of Thermal Expansion.

\section{LASER BASED THERMAL PROPERTY MEASUREMENT}

A major investment in research over the past 5 years has been the development of a laser based thermal property measurement capability. The TCM was demonstrated on the laser bench, which is a new measurement method that simultaneously measures local thermal diffusivity and conductivity. This was accomplished by measuring the temperature field spatial profile of samples excited by an amplitude modulated laser beam. A thin film is applied to the samples to ensure strong optical absorption and to establish a second boundary condition that introduces an expression containing the substrate thermal conductivity. The diffusivity and conductivity are obtained by comparing the measured phase profile of the temperature field to a continuum-based model.

Proof of principle studies were conducted on a range of samples having thermal properties that are representative of current and advanced ATF [2]. Measurements of conductivity and diffusivity for the lower conductivity samples agree closely with literature values. The moderate agreement for the high conductivity sample is attributed to neglecting the influence of Kapitza resistance between the film and the sample. The Kapitza resistance, $R_{t h}$, was estimated by fitting the continuum model to experimental results using literature values for the conductivity and diffusivity. The values of Rth for all of the samples investigated were in the range of $10^{-8} \mathrm{~m}^{2} \mathrm{~K} / \mathrm{W}$.

Additional measurements were made on three prototypic nuclear fuel surrogate samples: (1) depleted uranium alloyed with zirconium $\left(\mathrm{DU}_{80} \mathrm{Zr}\right.$ ), (2) depleted uranium oxide (stoichiometric $\mathrm{UO}_{2}$ ), and (3) depleted uranium silicide $\left(\mathrm{U}_{3} \mathrm{Si}_{2}\right)$. The $\mathrm{DU}_{80} \mathrm{Zr}$ and $\mathrm{UO}_{2}$ samples were also measured using laser flash. The thermal diffusivity obtained with the TCM compared favorably with laser flash measurements.

The uranium silicide sample was fabricated and prepared by Jason Harp and Alexander Winston at INL. The final polish of $0.25 \mu \mathrm{m}$ was performed by hand with medium force for roughly 5 minutes. As noted below, half of the sample was coated with a thin gold film and the other half was coated with a thicker gold film. 
As a method for gauging the influence of $R_{t h}$ for our silicide sample, the possibility was explored of extracting only the substrate diffusivity, $D_{s}$, using very thin films. Modeling results suggested that the thermal wave response should only be a function of $D_{s}$ for films thinner than $10 \mathrm{~nm}$. However this film thickness only corresponds to a fraction of the optical penetration depth. Consequently very little pump light is absorbed. As a compromise we took measurements on a sample having a film thickness corresponding to several optical skin depths $(\sim 40 \mathrm{~nm})$. For this case, modeling results show that there should be little sensitivity to substrate conductivity, $k_{s}$, only moderate sensitivity to $R_{t h}$ and good sensitivity to $D_{s}$. The extracted value for $D_{s}$ is $3.07 \times 10^{-6} \mathrm{~m}^{2} / \mathrm{s}$. This values compares favorably with the literature value at room temperature. Now that the value of $D_{s}$ is fixed we fit $k_{s}$ and $R_{t h}$ using the thicker film (thickness $\sim 160 \mathrm{~nm}$ ). The thicker film has optimal sensitivity to $k_{s}$. The experimental results corresponding to the thick film are presented in Figure 7. The extracted value of $R_{\text {th }}$ is $6 \times 10^{-6} \mathrm{~m}^{2} \mathrm{~K} / \mathrm{W}$ and the value of $k_{s}$ is $8.9 \mathrm{~W} / \mathrm{mK}$. This conductivity value also agrees favorably with literature values at room temperature.
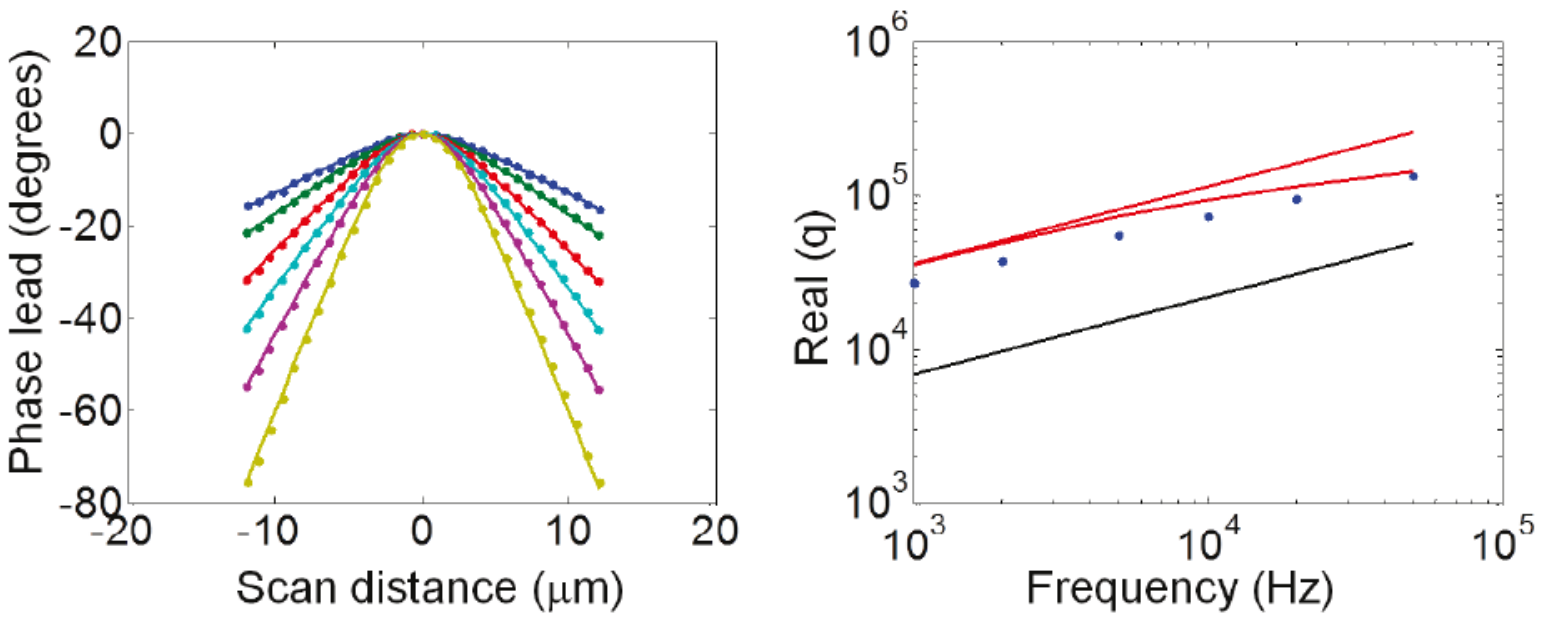

Figure 7. Left: Best fit results for $\mathrm{DU}_{3} \mathrm{Si}_{2}$ sample. Dots correspond to data and solid lines correspond to best fit modeling results. Right: Slope of the phase profile (q) in the far field of the source versus frequency. There is good sensitivity to both $k_{s}$ and $D_{s}$ for the frequency range investigated.

\section{PHYSICS OF THERMAL PROPERTY BEHAVIOR}

Nuclear fuel operates at high to very high temperatures and thermal conductivity and other materials properties lack sensitivity to temperature and material variations at reactor temperatures. Understanding the physics controlling the behavior of thermal properties of materials especially under irradiation is critical to understanding the thermal properties in general. Both the uncertainties in laboratory measurements at high temperatures and the small differences in properties of different materials will inevitably lead to large uncertainties in models and little predictive power. Conversely, properties measured at low to moderate temperature have more sensitivity (more variation in property with temperature), have less uncertainty, and have larger differences in properties for different materials. It is precisely these variations that need to be characterized, because if these property variations can be captured with high fidelity, they will afford the highest predictive capability in modeling and best assurances for validation and verification at all temperatures. The experimental work planned will be directly compared with NEAMS-funded modeling and simulations results provide us with exciting opportunity for reliable and successful code validation.

A key approach is to leverage cutting edge new experimental techniques and data that can be obtained at INL. Recent experimental characterization of the thermal conductivity of $\mathrm{UO}_{2}$ performed below room temperature has provided exciting insight into $\mathrm{UO}_{2}$ single crystal thermal conductivity [3]. It has been 
shown that resonance spin-phonon scattering can significantly reduce the thermal conductivity in this material and its couplings can cause a macroscopic anisotropic behavior.

The installation of the new experimental setup, the Quantum Design DynaCool-9 Physical Property Measurement System (PPMS) has been completed. This state-of-the-art experimental platform is design to measure various physical properties of small radioactive samples, including thermal conductivity. The INL's PPMS device is located in IRC-603, lab C6. In its current configuration, the setup allows the heat capacity, electrical resistivity, thermoelectric power, and thermal conductivity measurements in wide temperature (2-400 K) and magnetic field ranges (0-9 T). In the DynaCool device the thermal conductivity can be obtained by both direct steady state methods and by transient pulsed power (Maldonado) technique.

This new experimental capability, shown in Figure 8, will allow extensive and systematic transport, thermal, and thermodynamic studies of several fuel materials including $\mathrm{U}_{3} \mathrm{Si}_{2}, \mathrm{UO}_{2}, \mathrm{UN}$, and nonstoichiometric $\mathrm{UO}_{2+\mathrm{x}}$ crystals. The experimental data generated will allow comparison to predictions of the thermal conductivity from MD simulations existing in literature as well as to new simulations. The relative impact of various thermal conductivity contributions and scattering mechanisms observed in nuclear materials can be assessed. Simulations can also be on how they capture these processes.

Using the new experimental capabilities, preliminary thermal conductivity measurements were conducted on the $\mathrm{U}_{3} \mathrm{Si}_{2}$ polycrystalline sample used by Hurley and Papesch in the bulk and microscale thermal property measurements. The results are shown in Figure 9. It is note-worthy that noting that to our best knowledge these are the first thermal conductivity measurements for this material performed below room temperature. In general the thermal transport of $\mathrm{U}_{3} \mathrm{Si}_{2}$ is governed by phonon (heat transport by lattice vibrations) and electronic contributions. To separate the contributions and analyze the interactions of different scattering mechanisms (such as defects, grain boundary and phonon-phonon scattering) the electrical resistivity on this sample will be measured in future work at the INL.

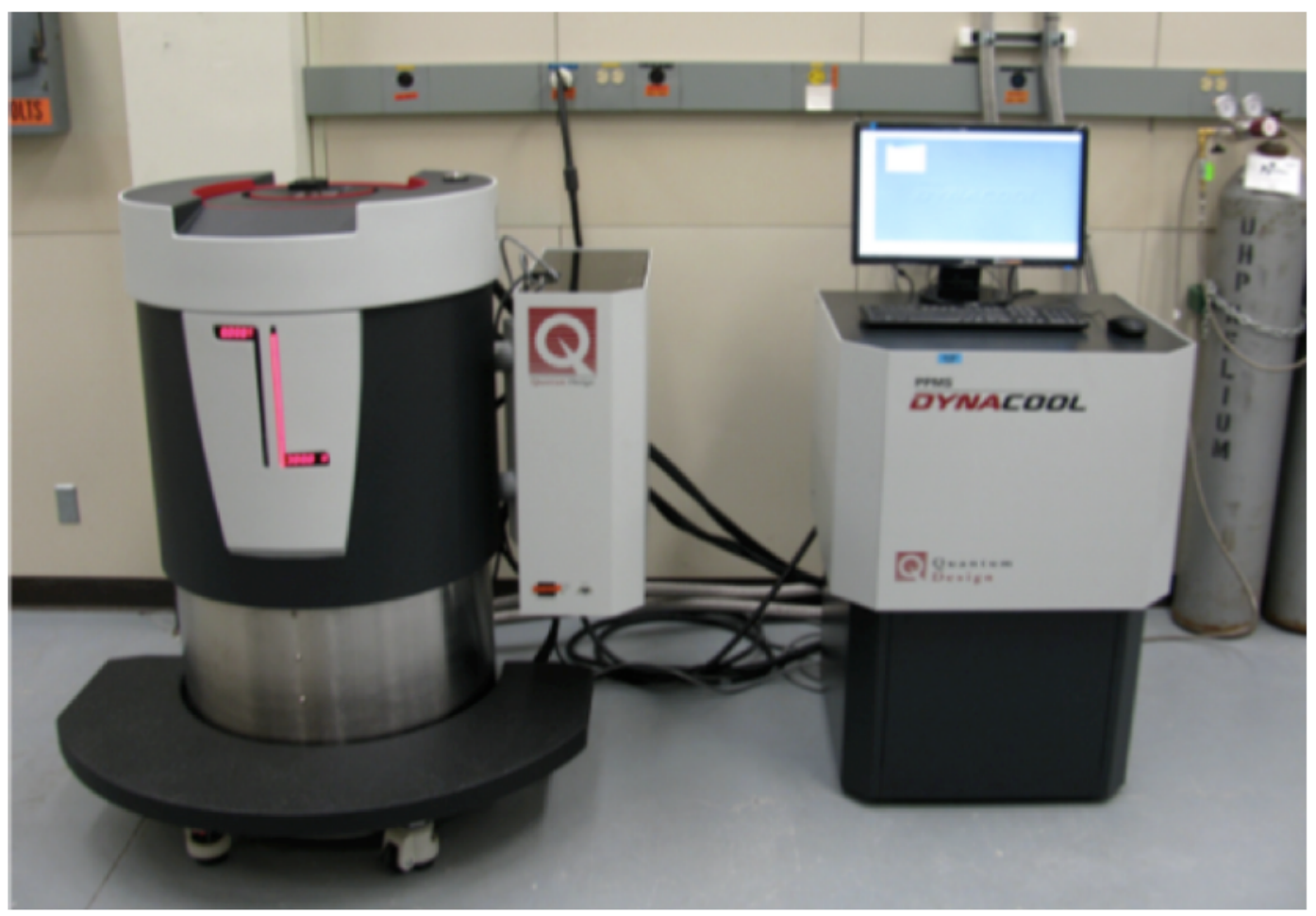

Figure 8. Quantum Design DynaCool-9 measurement system (Idaho Research Center, IF-603, C6). This closed circle measurement platform allows variety of transport and thermodynamic measurements of nuclear materials in wide temperature and magnetic field ranges. 


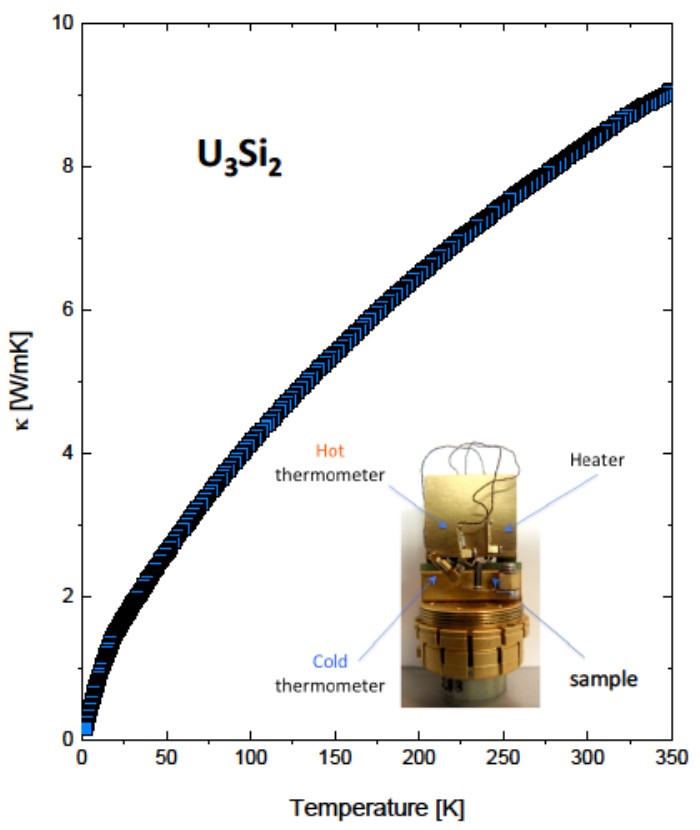

Figure 9. Total thermal conductivity measured on bulk $\mathrm{U}_{3} \mathrm{Si}_{2}$ sample in the range from $2 \mathrm{~K}$ to $350 \mathrm{~K}$. Inset: experimental setup used for the thermal conductivity measurements in the DynaCool system.

\section{MARMOT- MICROSTRUCTURE EVALUATION OF THERMAL CONDUCTIVITY OF $\mathrm{U}_{3} \mathrm{SI}_{2}$}

As described earlier, the new capabilities to measure the thermal conductivities of a wide range of fuels include the TCM for measurements at the microscale $(\sim \mu \mathrm{m})$, standard laser flash for measurements on bulk scale samples, and the physical properties measurement system (PPMS) recently established by Gofryk. These two methods have been applied to several types of fuels including $\mathrm{U}_{3} \mathrm{Si}_{2}$, a promising ATF candidate fuel. The measured thermal conductivity for a $\mathrm{U}_{3} \mathrm{Si}_{2}$ fuel sample recently fabricated at INL by the TCM method and standard laser flash differed from each other slightly. This difference suggests the impact of microstructure on the measurement, since these two methods work at difference spatial scales. The TCM method measures the conductivity and diffusivity locally (at $\sim 50 \mu \mathrm{m}$ resolution) for the $\mathrm{U}_{3} \mathrm{Si}_{2}$ matrix, while the laser flash method measures the overall bulk conductivity of the fuel, which consists of more complex microstructure.

Modeling using the MARMOT code [4] was conducted to demonstrate the ability to calculate the conductivity of a bulk material sample starting with the conductivity measured at the microstructural level. The MARMOT code is a powerful tool for calculating the effective thermo-physical properties of material systems with complex microstructures. This activity demonstrates a new science-based approach to fuels development by providing the ability to predict thermal properties of bulk materials in regimes and in samples beyond the capability of experimental method.

The fabricated $\mathrm{U}_{3} \mathrm{Si}_{2}$ fuel is not of a pure phase. Various phases other than the $\mathrm{U}_{3} \mathrm{Si}_{2}$ matrix have been identified, as indicated by contrast in the SEM image. Analysis of the chemical elements indicates that in addition to the $\mathrm{U}_{3} \mathrm{Si}_{2}$ matrix, two other phases may also exist: USi and an oxide phase, either $\mathrm{U}_{3} \mathrm{O}_{8}$ or hyper-stoichiometric $\mathrm{UO}_{2}$ to be cleared in the future by further characterization (Figure 10a). Here the modeling focuses on the impact of the oxide phase which has a much lower conductivity than those of the U-Si phases. In the model, the oxide phase is distinguished from the rest by reconstructing the SEM image using the image-processing tool in MOOSE (Figure 10b). Mesh adaptivity has been enabled at the interface. 
Based on the reconstructed mesh, the MARMOT code was used to calculate the effective thermal conductivity at room temperature. For the $\mathrm{U}_{3} \mathrm{Si}_{2}$ matrix, the value of $8.9 \mathrm{~W} / \mathrm{m} \cdot \mathrm{K}$ was obtained from the TCM measurement. For the oxide phase, two calculations were performed by taking the phase to be either $\mathrm{U}_{3} \mathrm{O}_{8}$ or hyper-stoichiometric $\mathrm{UO}_{2}$. A value of $2.2 \mathrm{~W} / \mathrm{m} \cdot \mathrm{K}$ from literature [5] was used for $\mathrm{U}_{3} \mathrm{O}_{8}$, and that of $4.3 \mathrm{~W} / \mathrm{m} \cdot \mathrm{K}[6]$ was used for $\mathrm{UO}_{2}$. The results show that the discrepancy between the results from TCM and laser flash measurements can be explained (Figure 10c) by considering the impact of the microstructure of the sample, i.e., the appearance of the oxide phase. The oxide phases have lower densities $\left(\sim 8.30 \mathrm{~g} / \mathrm{cm}^{3}\right.$ for $\mathrm{U}_{3} \mathrm{O}_{8}$ and $\sim 10.96 \mathrm{~g} / \mathrm{cm}^{3}$ for $\left.\mathrm{UO}_{2}\right)$ and thermal conductivities than those of $\mathrm{U}_{3} \mathrm{Si}_{2}$. Therefore, the presence of oxide phases leads to decreases in both mass density and thermal conductivity. Note that here the effect of USi phase has not been included in the calculation due to the lack of data for this phase. Better comparisons can be achieved in the future with further consideration of the microstructural details. Figure 10c also provides presentation of literature values using the thermal flash technique for comparison with the thermal conductivity data measured by Hurley, Papesch, and Gofryk on the same $\mathrm{U}_{3} \mathrm{Si}_{2}$ sample in the temperature range extending from $2 \mathrm{~K}$ to $900 \mathrm{~K}$. This is the first time this type of measurement has been performed using multiple measurement techniques for thermal conductivity and is only available at the INL for nuclear materials.

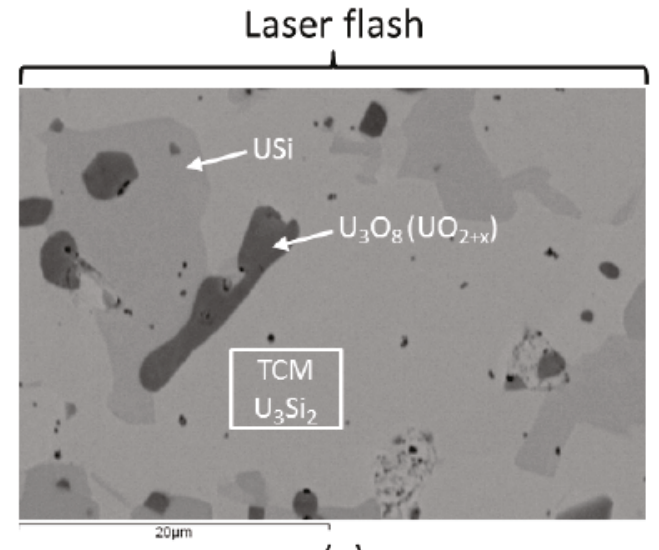

(a)

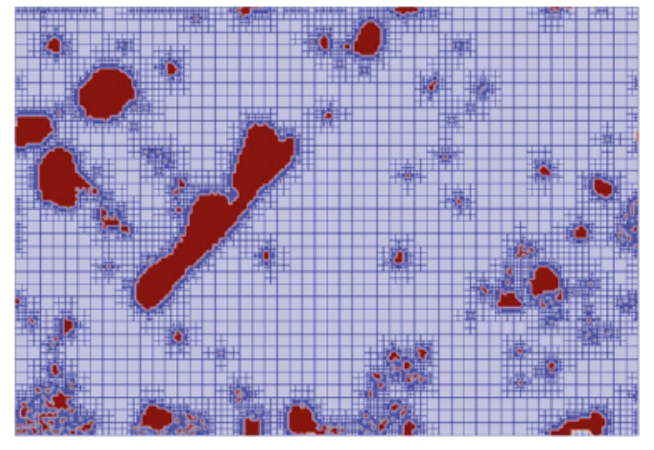

(b)

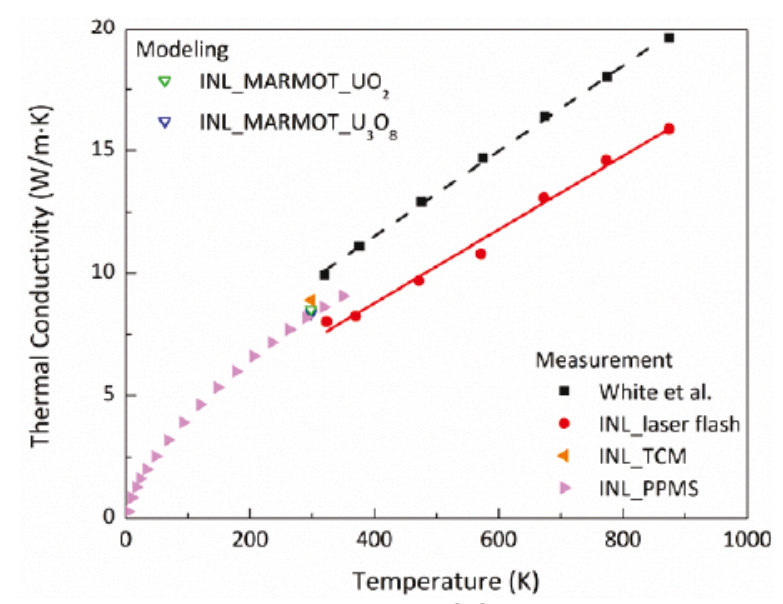

(c)

Figure 10. (a) SEM image of a $\mathrm{U}_{3} \mathrm{Si}_{2}$ sample, (b) reconstructed microstructure and mesh in MOOSE for MARMOT calculations, and (c) thermal conductivity of U3Si2 as a function of temperature. The solids symbols are experimental measurements from literature, INL Thermal Flash, INL TCM, and INL PPMS experimental as indicated. The MARMOT results based on the reconstructed mesh are represented by the open triangles at $300 \mathrm{~K}$. 


\section{IMCL THERMAL PROPERTY MEASUREMENT CAPABILITY STRATEGY}

A Thermal Properties Capability Development Workshop was organized at INL on April 14 to develop the strategy for thermal properties capability development at INL and to identify the potential suite of thermal property measurement equipment for IMCL [7]. Participants were encouraged to think broadly about their needs for thermal property measurements on a wide range of applicable fuels and materials. There are many philosophies and approaches to thermal property measurements, and the goal was to bring all those ideas together to decide how to push the science forward.

The recommended path forward from the workshop is to populate the IMCL's Thermal Properties Cell with equipment that can measure both bulk and microstructure properties of irradiated samples. The thermal properties measurement capability should be commensurate with the current thermal properties characterization line in the Fresh Fuels Glovebox. In addition, install a reflective-based measurement apparatus similar to the TCM, in the IMCL. This will provide the capability to do traditional thermal flash measurements, as well as using the TCM to measure samples that cannot be prepared appropriately for the thermal flash measurement technique. Combination of techniques implemented at different length scales allows fundamental understanding of the effect of microstructure on thermal transport.

\section{LEADERSHIP AND AWARDS}

INL researcher Krzysztof Gofryk was selected as one of 44 scientists to receive significant funding for research as part of DOE's Office of Science Early Career Research Program. Recently, he has gained recognition for his research in actinide materials under extreme conditions and will receive funding support from this program during the next five years. Patricia Dehmer, acting director of DOE's Office of Science, noted, "Supporting talented researchers in their early career years is one key to building and maintaining an effective scientific workforce for the nation." At the INL annual recognition event, Gofryk was also recognized with an award for Outstanding Paper by Early Career Scientist or Engineer by publishing, "Anisotropic Thermal Conductivity in Uranium Dioxide," in Nature Communications. 


\section{REFERENCES}

1. J.T. White, A.T. Nelson, D.D. Byler, D.J. Safarik, J.T. Dunwoody, K.J. McClellan, "Thermophysical Properties of U3Si5 to 1773 K," Journal of Nuclear Materials, Vol 456, January 2015, Pages 442448.

2. D. Hurley, R. Schley, "Status Report on the Development of the Thermal Conductivity Microscope," 2015. INL/LTD-15-35366.

3. K. Gofryk et.al, "Anisotropic Thermal Conductivity in Uranium Dioxide," Nature Communications, 2014.

4. M.R. Tonks, D. Gaston, P.C. Millett, D. Andrs, and P. Talbot, "An Object-Oriented Finite Element Framework for Multiphysics Phase Field Simulations," Computational Materials Science 51 (2012) 20-29.

5. C.G.S. Pillai, A.K. Dua, and P. Raj, "Thermal Conductivity of $\mathrm{U}_{3} \mathrm{O}_{8}$ from 300 to $1100 \mathrm{~K}$," Journal of Nuclear Materials, 288 (2001) 87-91.

6. P.G. Lucuta, Hj. Matzke, R.A. Verall, "Thermal Conductivity of Hyperstoichiometric SIMFUEL," Journal of Nuclear Materials, 223 (1995) 51-60.

7. L. Braase, C. Papesch, "Thermal Properties Capability Development Workshop Summary to Support the Implementation Plan for PIE Thermal Conductivity Measurements," April 2015. FCRD-FUEL2015-000219. INL/EXT-15-35125.

Please contact J. Carmack for further information regarding this report. 